\title{
PROBLEMAS FITOSANITARIOS QUE AMENAZAN LA CONSERVACIÓN DE LAS ORQUÍDEAS EN COSTA RICA
}

\author{
German Rivera-Coto $^{1,3} \&$ Gilberto Corrales-Moreira $^{2}$ \\ ${ }^{1}$ Laboratorio de Fitopatología , Universidad Nacional, Apartado Postal 86-3000, Heredia, Costa Rica. \\ ${ }^{2}$ Laboratorio de Entomología, Universidad Nacional, Apartado postal 86-3000, Heredia. Costa Rica. \\ ${ }^{3}$ Autor para correspondencia: grivera@una.ac.cr
}

\begin{abstract}
Orchids from private collections, nurseries and wild areas, located in different ecological regions of Costa Rica, were studied in order to know the pests and diseases affecting them. 16 insect and two mite genera were identified as pests on different orchids and 28 pathogens were found as causal agents of infectious diseases. The most frequent pests were: Tenthecoris orchidearum, Stethobaris sp., Xylosandrus compactus, Pseudococcus longispinus, Diaspis boisduvalii and Tenuipalpus pacificus. The main diseases found were caused by: Phytophthora spp., Pythium spp., Fusariun oxysporum, Fusarium spp., Sclerotium rolfsii, Colletotrichum gloeosporioides, Sphenospora spp., Uredo spp., Cymbidium Mosaic virus and Odontoglossum Ring Spot Virus. This results demonstrate the diversity of organisms producing sanitary problems on orchids in Costa Rica, and suggests the inclusion of pest and diseases management in strategies for orchid conservation.
\end{abstract}

KEY WORDS: orchid diseases, orchid pests, enfermedades, plagas, sanidad vegetal

\section{Introducción}

La conservación de las orquídeas debe ser una acción integral donde participen especialistas de diferentes disciplinas, sin olvidar la meta común de proteger esta noble familia de plantas. La pérdida de especies no es producto de un solo factor, es por el contrario, el efecto combinado de varios de ellos, particularmente los que son manipulados por el hombre. Dentro de estos debe destacarse la modificación que hemos hecho en el entorno biótico y abiótico, la extracción y comercialización ilegal de plantas, el mal manejo cultural de las orquídeas y los problemas sanitarios.

Las iniciativas para poner en marcha programas de conservación y rescate de especies, así como la protección de extensas regiones donde estas plantas viven y se reproducen libremente. Han hecho una función muy importante y se han obtenido avances significativos, en lo que a conservación se refiere. Sin embargo, algunas veces el resultado de todo ese esfuerzo y recursos puede verse amenazado por factores poco perceptibles en un inicio, pero devastadores a mediano o largo plazo, como son los problemas fitosanitarios que afectan las orquídeas.

Por lo general, las especies conservadas in situ no son tan amenazadas por plagas microbianas o de artrópodos, pues en la naturaleza existen controladores bioló- gicos y diversas interacciones en el ecosistema que mantienen el problema bajo control, excepto cuando llegan organismos exóticos sin sus antagonistas. En las colecciones ex situ el panorama es diferente debido a que la intrincada red de interacciones, común en ecosistemas no alterados, está ausente o incompleta, las condiciones de crecimiento son artificiales, los genotipos son más homogéneos y hay una mayor manipulación tanto física como genética de las plantas. Esto hace que las plagas puedan encontrar un substrato muy propicio para desarrollarse y poner en peligro la sobrevivencia de especies muy selectas, escasas o ausentes en estado silvestre. Por lo tanto, sea cual fuere la modalidad de conservación siempre existe el peligro real o potencial de problemas fitosanitarios. Partiendo de esta premisa, la Escuela de Ciencias Agrarias de la Universidad Nacional, tomó por iniciativa propia el objetivo de estudiar los problemas sanitarios de las orquídeas en Costa Rica, con el fin de contribuir a la conservación de estas plantas en los diferentes sitios donde crecen cultivadas o en condición silvestre, como son las colecciones privadas, viveros, jardines botánicos, bosques cercanos a sitios de cultivo o áreas protegidas. Con este estudio se pretende establecer una plataforma de partida para proteger en una forma más integral las orquídeas de 
Costa Rica y establecer los nexos necesarios con otros profesionales comprometidos con la conservación de las orquídeas.

\section{Metodología de trabajo}

Esta investigación dio inicio en el 2003 y concluirá en diciembre del 2006. Para su ejecución se consideró las diferentes regiones del país y se planificó una serie de visitas a cada una de ellas, iniciándose primero con el Pacífico Sur y Pacífico Central, luego se continuó con el Caribe y Zona Norte, para cerrar el proceso en el Pacífico Norte y el Valle Central. En cada región se contactó las diversas asociaciones regionales de orquideología, grupos de orquideófilos, productores comerciales y centros de investigación en este campo. Estos grupos o instituciones brindaron su ayuda en la ubicación de los sitios de visita y recorridos. A cada visita asistió un entomólogo y un fitopatólogo para hacer un reconocimiento de los problemas presentes, condiciones del sitio, historial sanitario y también para realizar la toma de muestras de plantas o partes de ellas con síntomas de problemas sanitarios. Los materiales recolectados fueron llevados a los respectivos laboratorios en la Universidad Nacional, para hacer los diagnósticos. En aquellos sitios donde habían orquídeas creciendo en condición silvestre o semisilvestre en fincas o bosques de los alrededores, también se tomaron muestras para estudio. En el laboratorio se realizaron los análisis rutinarios para organismos patógenos, insectos y ácaros y se levantó un registro de resultados e imágenes fotográficas. Cada vez que se concluyó el trabajo en una región o comunidad se brindó una conferencia sobre los resultados obtenidos y se brindaron las recomendaciones pertinentes para el manejo de esos problemas.

\section{Resultados}

Los patógenos observados hasta el momento en este estudio son 18 géneros de hongos, dos de Oomycetes, dos de Mixomycetes, cuatro bacterias, un alga y dos virus. Los hongos fitopatógenos identificados en diversos géneros de orquídeas corresponden a los siguientes:

Botrytis cinerea Pers.: Fr., agente causal de lesiones en flores de los siguientes géneros: Cattleya,
Epidendrum, Gongora, Guarianthe, Miltonia, Phalaenopsis, Peristeria, Pescatorea, Psychopsis, Stanhopea, Vanda y Xylobium.

Capnodium sp. asociado a fumaginas en: Dendrobium, Encyclia, Gongora, Maxilaria, Phalaenopsis, Pleurothallis, Rodriguezia, Stanhopea y Vanda.

Cercospora spp. presente en manchas foliares de los géneros: Acineta, Aspasia, Brassia, Calanthe, Catasetum, Cattleya, Coelogyne, Dendrobium, Dendrochilum, Encyclia, Epidendrum, Gongora, Huntleya, Lycaste, Maxilaria, Mormodes, Oncidium, Phaius, Pleurothallis, Rossioglossum, Sarcoglottis, Stanhopea y Trichopilia.

Cercosporidium sp. causando manchas difusas en el follaje de: Cattleya, Encyclia, Lycaste, Stanhopea y Trigonidium.

Cladosporium sp., desarrollando fumagina en las inflorescencias de Arundina graminifolia (Don) Hochr.

Colletotrichum gloeosporioides (Penz.) Penz \& Sacc [Glomerella cingulata (Stoneman) Spauld \& Schrenk.], causando antracnosis en los siguientes géneros: Acineta, Angraecum, Arundina, Aspasia, Brassavola, Brassia, Calanthe, Catasetum, Cattleya, Caularthron, Chysis, Cochleanthes, Coelogyne, Coryanthes, Cycnoches, Cymbidium, Dendrobium, Dracula, Dressleria, Encyclia, Epidendrum, Gongora, Guarianthe, Huntleya, Laelia, Lockhartia, Lycaste, Masdevallia, Maxilaria, Miltonia, Mormodes, Notylia, Oncidium, Paphiopedilum, Peristeria, Pescatorea, Phaius, Phalaenopsis, Pleurothallis, Polystachya, Psychopsis, Rossioglossum, Scaphyglottis, Schombugkia, Sobralia, Stanhopea, Trichopilia, Trigonidium, Vanda, Vanilla y Xylobium.

Fusarium oxysporum Schlechtend.:Fr., como agente causal de marchitez en: Bulbophyllum, Calanthe, Cattleya, Clowesia, Coelogyne, Cymbidium, Dendrobium, Encyclia, Epidendrum, Gongora, Guarianthe, Laelia, Lemboglossum, Miltonia, Mormodes, Oncidium, Phalaenopsis, Rodriguezia, Rossioglossum, Scaphyglottis, Vanilla y Xylobium.

Fusarium spp., este patógeno fue aislado en diversas ocasiones a partir de pudriciones en raíces, rizomas y seudobulbos de géneros como: Acineta, Brassia, Catasetum, Cattleya, Coelogyne, Dendrobium, 
Encyclia, Gongora, Guarianthe, Laelia, Lycaste, Mormodes, Vanilla y Xylobium.

Graphium sp., este hongo fue identificado en cultivos puros obtenidos a partir de manchas foliares en: Cattleya, Cochleanthes, Coelogyne, Guarianthe, Lycaste y Oncidium.

Guignardia sp., se observó como agente causal de manchas foliares en Ascocentrum y Vanda.

Lasiodiplodia (Botryodiplodia) sp., hongo asociado con diversas manchas en: Brassia, Cattleya, Elleanthus, Gongora, Guarianthe, Maxilaria, Oncidium, Psychopsis, Sobralia, Stanhopea, Vanda y Vanilla.

Meliola sp., agente causal del mildiú negro en: Dendrobium, Dressleria, Schomburgkia y Vanilla.

Mycoleptodiscus sp., causante de manchas foliares en los siguientes géneros: Brassia, Coelogyne, Cyrtopodium, Elleanthus, Maxilaria, Oncidium, Phaius, Pleurothallis, Sobralia, Stanhopea y Xylobium.

Phyllosticta sp., asociado a manchas foliares en: Aspasia, Encyclia, Epidendrum y Vanilla.

Sclerotium rolfsi Sacc., agente causal de pudriciones en la raíz de Phalaenopsis y base de la planta en Spathoglottis.

Sphenospora spp., causante de la roya en: Catasetum, Encyclia, Epidendrum y Stanhopea.

Schyzothirium sp., patógeno hallado en manchas foliares de los géneros: Elleanthus, Oncidium, Pleurothallis, Sobralia y Stanhopea.

Uredo sp. agente causal de royas en: Brassavola, Cattleya, Encyclia, Epidendrum, Laelia, Pleurothallis y Trigonidium.

Los restantes patógenos (Oomycetes, bacterias, Myxomycetes, algas y virus) identificados en diferentes localidades del país se presentan en el cuadro 1, con sus respectivos hospederos. De ese grupo destacan por su frecuencia los Oomycetes, causantes de la pudrición negra de plántulas y plantas adultas, la bacteria Erwinia, agente causal de pudriciones suaves y los virus que propician el desarrollo de síntomas como anillos cloróticos, anillos necróticos, estrías foliares y variegado de flores.

En el cuadro 2 se presentan los artrópodos determinados como causantes de daños en orquídeas cultivadas y silvestres. En términos generales puede señalar- se que se logró observar cuatro coleópteros, un hemíptero, siete homópteros, un lepidóptero, un ortóptero, dos himenópteros y dos ácaros, asociados a daños en orquídeas de diversos géneros y colectadas en distintas partes del país.

\section{Discusión}

En Costa Rica hay una amplia diversidad de orquídeas, de la cual aún no se conoce la totalidad de especies (Morales 2005a, Morales 2005b), de igual manera, existe un número considerable de organismos que atacan las orquídeas, de los cuales tampoco se tiene un inventario completo en el país. Los resultados de esta investigación reflejan esa diversidad de organismos tanto autóctonos como exóticos, distribuidos en todas las localidades visitadas (Rivera \& Corrales 2003, Corrales \& Rivera 2003, Rivera \& Corrales 2005). Por ser este el primer intento por censar los problemas fitosanitarios, es posible que haya más organismos en otros sitios y se requiera más años de trabajo, para completar la lista de patógenos y artrópodos potencialmente capaces de convertirse en plaga.

En las enfermedades de orquídeas determinadas hasta el momento, en el territorio nacional, se puede hablar de tres grandes categorías de enfermedades: letales, degenerativas y las de impacto reducido. En el primer grupo están las que liquidan la planta en un tiempo relativamente corto, las cuales se convierten en la principal amenaza para la conservación de especies muy valiosas. Los patógenos que pueden ubicarse en esa categoría son: Phytophthora spp., Pythium spp., Erwinia spp., Fusarium spp. y $S$. rolfsii. Los dos primeros son los agentes causales de la pudrición negra en rizomas y seudobulbos, su acción es muy rápida y en pocos días elimina un espécimen ( Cárdenas 2003); La bacteria Erwinia causa pudriciones suaves en rizomas y seudobulbos, en ellos desintegra todos los tejidos blandos con rapidez; S. rolfsii y Fusarium spp. son causantes de pudriciones masivas del tejido radical y causan a corto plazo la muerte de las plantas afectadas, al limitarse la absorción de agua y minerales.

La segunda categoría incluye las enfermedades que eliminan el hospedero en forma paulatina y sostenida propiciando un decaimiento progresivo hasta causar la 
CUADRO 1. Oomycetes, bacterias, Mixomycetes, algas y virus identificados en plantas enfermas de orquídeas procedentes de distintas zonas ecológicas de Costa Rica.

\begin{tabular}{|c|c|}
\hline Patógeno & Nombre de los géneros de orquídeas hospederas \\
\hline \multicolumn{2}{|l|}{ OOMYCETES } \\
\hline Phytophthora spp. & $\begin{array}{l}\text { Cattleya, Cymbidium, Dendrobium, Dracula, Encyclia, Epidendrum, Guarianthe, Lockhartia, } \\
\text { Lycaste, Masdevallia, Maxilaria, Oncidium, Rodriguezia, Scaphyglottis, Schomburgkia, Sobralia. }\end{array}$ \\
\hline Pythium spp. & $\begin{array}{l}\text { Cattleya, Coelogyne, Dendrobioum, Guarianthe, Masdevallia, Pescatorea, Phalaenopsis, } \\
\text { Pleurothallis, Vanda. }\end{array}$ \\
\hline \multicolumn{2}{|l|}{ BACTERIAS } \\
\hline Erwinia spp. & $\begin{array}{l}\text { Brassia, Calanthe, Catasetum, Cattleya, Coelogyne, Cyrtopodium, Dendrobium, Guarianthe, } \\
\text { Lycaste, Maxilaria, Miltonia, Oncidium, Phaius, Phragmipedium, Schomburgkia, Stanhopea, } \\
\text { Trichopilia, Vanda. }\end{array}$ \\
\hline Pantoea sp. & $\begin{array}{l}\text { Brassia, Catasetum, Cattleya, Epidendrum, Guarianthe, Laelia, Maxilaria, Oncidium, Pescatorea, } \\
\text { Phalaenopsis. }\end{array}$ \\
\hline Xanthomonas sp. & Guarianthe, Schomburgkia, Vanilla. \\
\hline Pseudomonas sp. & Coelogyne, Phalaenopsis, Rossioglossum. \\
\hline \multicolumn{2}{|l|}{ MIXOMYCETES } \\
\hline Physarum sp. & Guarianthe, Oncidium, Trichopilia. \\
\hline Hemitrichia sp. & Trichopilia. \\
\hline \multicolumn{2}{|l|}{ Algas } \\
\hline Cephaleuros virescens Kunze & Cattleya, Coelogyne, Elleanthus, Masdevallia, Oncidium, Sobralia. \\
\hline \multicolumn{2}{|l|}{ VIRUS } \\
\hline ORSV-TMVO & $\begin{array}{l}\text { Acineta, Cattleya, Cochleanthes, Dendrobium, Encyclia, Epidendrum, Guarianthe, Schomburgkia, } \\
\text { Sobralia, Trichopilia, Vanda. }\end{array}$ \\
\hline CyMV & Cattleya, Cymbidium, Encyclia, Lycaste, Oncidium, Phaius, Trichopilia. \\
\hline
\end{tabular}

muerte. En este grupo hay varios patógenos como: $C$. gloeosporioides, F. oxysporum, virus del mosaico del Cymbidium (CyMV), virus del anillado del Odontoglossum (ORSV) y varias royas. El hongo $C$. gloeosporioides es el agente causal de la antracnosis, la cual es considerada como la más frecuente en los trópicos (AOS 1995, Rivera 1998) y llega a ser una enfermedad degenerativa en plantas mal nutridas o en estado de abandono; $F$. oxysporum actúa como un patógeno vascular que lentamente bloquea la circulación de savia en la planta, por lo tanto, origina una serie de síntomas reflejos en hojas y tallos como deshidratación, cambios de color y enanismo, entre otros; los virus por sus características patogénicas tan particulares son un buen ejemplo de muerte lenta y prolongada en muchas especies de orquídeas al declinar el vigor de la planta por periodos largos o en forma recurrente; para algunos géneros de orquídeas las royas constituyen un problema serio al reducir el crecimiento y propiciar una condición precaria por años.
El último grupo lo conforman las enfermedades de impacto reducido, las cuales en condiciones normales de manejo, no comprometen la supervivencia del hospedero. Sin embargo, bajo estrés constante o estado de abandono, pueden afectar considerablemente la salud de la planta y aniquilarla. A esta categoría pertenece la mayor parte de las enfermedades observadas en este estudio y algunas de ellas son: las manchas foliares, manchas en la flor, así como lesiones en los seudobulbos y tallos.

Entre los artrópodos asociados a daños en orquídeas destacan por su importancia como plagas los siguientes: Tenthecoris orchidearum Reuter, Stethobaris sp., Xylosandrus compactus Eichhoff, Pseudococus longispinus Targione-Tozzetti, Diaspis boisduvalii Signoret y Tenuipalpus pacificus Baker. De todos ellos T. orchidearum, conocido entre los cultivadores como chinche rojo, es el más frecuente. Los daños los causan principalmente las ninfas que se ubican en el envés de las hojas, donde provocan una decoloración 
CUADRo 2. Artrópodos causantes de daños en orquídeas identificados en distintas regiones de Costa Rica.

Identificación

Coleoptera: Curculionidae

Stethobaroides sp.

Stetobaris sp.

COleOptera: SCOLYTiDAe

Xylosanrdrus compactus Eichhoff

Brassia, Guarianthe, Laelia.

COleoptera: Mordellida

Mordillistema $\mathrm{sp.}$

Cattleya, Vanda.

Hemiptera: Miridae

Tenhtecoris orchidearum Reuter

Catasetum, Clowesia, Cattleya, Epidendrum, Encyclia, Gongora, Guarianthe,

Masdevallia, Maxillaria,Oncidium, Restrepia, Rodriguezia, Stanhopea,Stelis, Vanda.

HOMOPTERA: DIASPIDIDAE

Diaspis boisduvalii Signoret, Saisettia hemispherica Walker, Vinsonia stellifera

Westwood, Chrysomphalus sp.

Homoptera: Pseudococcidae

Pseudococcus longispinus Targione-

Tozzetti

Homoptera: ApHIDIDAE

Afidos aún no identificados

Cerataphis orchidearum Westwood

Brassavola, Cattleya, Encyclia, Epidendrum, Guarianthe, Phalaenopsis.

Acineta, Brassia, Cattleya, Encyclia, Epidendrum, Guarianthe, Laelia, Lockhartia, Oncidium, Isochilus.

LEPIDOPTERA: NoctUIDAE

Polilla aún no identificada

Brassia, Cattleya, Encyclia, Guarianthe, Laelia, Schomburgkia, Stanhopea.

HYMENOPTERA: Formicidae

\begin{tabular}{l|l}
\hline Atta sp. & Dendrobium, Sobralia.
\end{tabular}

HYMENOPTERA: EURYTOMIDAE

Avispas agalladoras no identificadas

Encyclia, Oerstedella.

Ortoptera: Tetigonidae

Idiarthron $\mathrm{sp}$.

Acineta, Brassavola, Brassia, Cattleya, Guarianthe, Miltonia, Mormodes, Phalaenopsis, Rossioglossum, Stanhopea, Trichopilia.

Acarina: Tenuipalpidae

Tenuipalpus pacificus Baker

Tetranichus sp.

Cymbidium, Dendrobium, Epidendrum, Phalaenopsis.

Dendrobium, Gongora, Phaius, Stanhopea.

blanquecina visible en ambos lados de la hoja. Las orquídeas con lámina foliar más delgada o moderadamente gruesa, son las que presentan mayor intensidad del daño. Esta plaga se ha observado en distintas regiones del país durante todo el año, pero hay una tendencia hacia el incremento de la población en periodos de escasa precipitación. El segundo insecto más frecuente fue la escama $D$. boisduvalii, la cual se encontró causando severos daños especialmente en viveros bajo techo de plástico o vidrio. Sin embargo, 
también se observó con menor frecuencia, en plantas ubicadas en sombreaderos o al aire libre.

Los restantes insectos aparecen con frecuencia pero como casos aislados, donde no se ha apreciado daños severos, lo cual no implica que sean de importancia secundaria, pues también pueden convertirse en plagas bajo determinadas condiciones.

Si se desea establecer un plan para proteger las orquídeas de este país, en forma integral y sostenible en el tiempo, es indispensable incluir aspectos fitosanitarios en él. Esta necesidad se hace aún más evidente al analizar los resultados de este trabajo, el cual muestra la diversidad de artrópodos y microorganismos como plagas reales o potenciales, que se convierten en una amenaza permanente para la conservación de las orquídeas en Costa Rica.

Agradecimientos. Se agradece a la Dirección de Investigación de la Universidad Nacional, por el apoyo financiero, al Jardín Botánico Lankester y a las asociaciones de orquideología de todo el país por la cooperación prestada en el trabajo de campo, así como a los coleccionistas y viveristas privados por permitirnos trabajar con sus plantas.

\section{LITERATURA CITADA}

American Orchid Society. 1995. Orchid pests and diseases.

Florida. Book Department Services. AOS. 88p.

Cárdenas, C. 2003. Estudio de la pudrición negra de las orquídeas causada por Phytophthora sp. en colecciones del Valle Central de Costa Rica. Lankesteriana 7: 179180.

Corrales, G. \& G. Rivera. 2003. Principales artrópodos asociados con colecciones y viveros de orquídeas en la región del Pacífico Sur de Costa Rica. V Congreso Nacional de Fitopatología. San José Costa Rica. 17 -1 9 de noviembre 2003. $54 \mathrm{pp}$.

Morales, J.F. 2005a. Orquídeas de Costa Rica. Vol. 1. Heredia, Costa Rica. Editorial INBio. 180 p.

Morales, J.F. 2005b. Orquídeas de Costa Rica. Vol. 2. Heredia, Costa Rica. Editorial INBio. 165 p.

Rivera, G. 1998. Orquídeas, generalidades y cultivo. Heredia, Costa Rica. Editorial Fundación UNA. 263 pp.

Rivera, G. \& G. Corrales. 2003. Principales enfermedades fungosas diagnosticadas en colecciones y viveros de orquídeas en la región del Pacífico Sur de Costa Rica. V Congreso Nacional de Fitopatología. San José Costa Rica.17 -1 9 de noviembre 2003. 27pp.

RRivera, G. \& G. Corrales. 2005. Enfermedades fungosas y bacterianas de las orquídeas cultivadas en distintas zonas ecológicas de Costa Rica. XLV Congreso Anual de la Sociedad Americana de Fitopatología- División Caribe. VI Congreso Nacional de Fitopatología. San José. 27 de junio-0 de julio del 2005. 88pp.

Salazar, W., G. Rivera \& G. Corrales. 2005. Identificación de patógenos e insectos que afectan la sanidad de las orquídeas epífitas en poblaciones silvestres de la zona protectora Cerros de la Carpintera. XLV Congreso Anual de la Sociedad Americana de Fitopatología- División Caribe. VI Congreso Nacional de Fitopatología. San José. 27 de junio-0 de julio del 2005. 88 pp.

German Rivera Coto. Catedrático de la Universidad Nacional. Ha trabajado por 30 años como Fitopatólogo en programas de investigación, docencia y extensión universitaria. En los últimos 20 años ha orientado su trabajo hacia la capacitación en el cultivo de las orquídeas y la investigación de los problemas fitopatológicos de la familia Orchideaceae, particularmente sobre enfermedades fungosas. Es autor de un libro sobre cultivo de las orquídeas con énfasis en los problemas sanitarios, varios artículos de revistas y ponencias en congresos nacionales e internacionales.

Gilberto Corrales Moreira. Profesor jubilado de la Universidad Nacional y de la Universidad de Costa Rica, donde fue docente, investigador y extensionista en entomología agrícola. En los últimos años ha colaborado ad honorem en el proyecto de investigación: Identificación de los patógenos y artrópodos que afectan la sanidad de las orquídeas en poblaciones silvestres y cultivadas. Producto de su trabajo ha realizado varias publicaciones sobre plagas de orquídeas. 